\title{
Smart urban farming service model with IoT based open platform
}

\author{
Am suk Oh \\ Department of Digital Media Engineering, Tongmyong University, South Korea
}

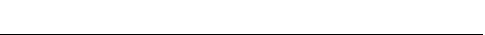

\section{Corresponding Author:}

Am suk Oh,

Department of Digital Media Engineering,

428 Sinseon-ro, Nam-gu, Busan, 48520, South Korea.

Email: asoh@tu.ac.kr

\begin{tabular}{l}
\hline \hline Article Info \\
\hline Article history: \\
Received Feb 11, 2020 \\
Revised Apr 12, 2020 \\
Accepted Apr 26, 2020 \\
\hline Keywords: \\
Cloud \\
Greenhouse standardization \\
Internet of things \\
Smart farm \\
Urban farming
\end{tabular}

\begin{abstract}
Smart and efficient agricultural production or smart farming using IoT sensors, big-data and cloud service has proven its value for a decade but the effect depends on the agricultural environment of the country or society. In Korea, the population of urban farmers who utilizes small, possibly shared area in farming. Urban farming uses rooftop of the building or even indoor for cropping and many urban farmers may not have sufficient professional farming experiences. However, with information technology like cloud service, many critical farming process can be automated and requires minimal human intervention in monitoring and controlling sensors. In this paper, we propose a smart urban farming model which modifies TTA smart greenhouse standard such that cloud service us integrated with IoT sensors. The hardware design of integrated controller and subsequent software services are specified. This new model can be used to enhance smart urban farming which is one of top 10 agricultural policy of the government.
\end{abstract}

Copyright $\left(C_{2} 2020\right.$ Institute of Advanced Engineering and Science. All rights reserved.

\section{INTRODUCTION}

Precision agriculture, a farming management with observing, measuring and responding to inter and intra-field variability in crops based on decision supporting system, has been adopted in recent 15 years [1]. With recent rapid development of Internet of things (IoT) technology [2], the process of real-time data gathering, processing and analysis become equally important as the traditional automation technology of the farming processes in precision agriculture [3]. This concept of smart farming extends the automation of farming toward more informed decision making by the farmers for better production of crops [4].

Smart farming based on IoT technology can reduce human interaction drastically to improve the efficiency of farming [5]. In the process of automation, IoT collects data using sensors and processes the data using controllers and completing the automation processes by using actuators [6]. It is reported that for all Australian farmers it became mandatory to affix passive RFID ear tags to their cattle and to report movements between farms to an online national database [7]. There have been some noticeable progresses and experiences in applying IoT for smart farming procedure with different agricultural environment in recent years [8-11] where they show partial successes and limitations.

Communication technology also plays an important role in smart farming. In [7], the Global Sensor Network (GSN) [12] middleware is employed, while summaries of sensor data are translated to resource description form, based on the Semantic Sensor Network (SSN) ontology [13]. Other researches use wireless sensor network [14] and/or low power Bluetooth and Low Power Wide Area Networks (LPWAN) and the monitoring and control functions are under the Message Queuing Telemetry Transport (MQTT) which is an IoT dedicated protocol [15], Overall, this trend of applying smart farming in agriculture is worldwide including South East Asia [16] and western Africa [17]. 
In Korea, smart farming becomes one of top ten national agricultural policy adopted by the Ministry for Food, Agriculture, Forestry and Fisheries (MFAFF). In [18], MFAFF plans to distribute smart farm facilities to 4,000 ha of facility horticulture (40\% of facility modernization), 730 livestock farms (10\% of full-time farmers) and 600 fruit trees (25\% of orchard-scale farms) by 2022 and expect artificial intelligence (AI) based control system that automatically learns and analyzes the growth and environmental information automatically acquired from IoT sensors.

However, in Korea, they have another farming issue called urban farming. Urban farming is the practice of cultivating, processing and distributing food in or around urban areas [19]. With rapid coming of high-aging society and youths' job crisis, if well designed as a policy, urban farming/agriculture could be a strategy for sustainable regional development, social safety net construction, and vocational training [20]. It is possible to participate without specialized skills in agriculture/farming through gradual education and also it is afford physically for young people or retired citizens [21].

Smart urban farming is then a much needed supporting technology for this socio-economic policy from government's point of view. Nonetheless, there is some negative result in policy implementation. MFAFF distributed stand-alone type smart farm equipment to 820 farms in 2015, but only 62 farmers were successful in evaluation, with only $7.6 \%$ success rate [22]. That is partly due to the aging of the rural population. Most farm owners are less willing to acquire knowledge about new technologies, which may limit their immediate response to instrumentation and partial system failures and failures. Also, there is some environmental difference from other countries. For example, while $99 \%$ of farmhouses adopt glass greenhouses in Netherlands, the majority (over 90\%) of farms in Korea depend on plastic greenhouses. Since plastic greenhouses are less durable and less productive than glass greenhouses, it is difficult to apply, disseminate and expand information technology in the field [23].

In this paper, we propose a model of smart urban farming based on open platform IoT technology. The system configuration of urban smart farm solution proposed in this paper composed of cloud service type through IoT service platform, which is different from existing integrated control system type. For this purpose, integrated functions of greenhouse integrated controller, sensor node, and control node of the existing system are integrated into the smart farm integrated controller (in this case, comply with the standard specification for interoperability (TTAK.KO-06.0288 [24]). Smart Farm Integrated Controller connects directly to Smart Farm service platform as an IoT device. The proposed model simplifies the configuration and structure of the entire system and provides unrestricted device connectivity.

\section{SMART URBAN FARMING SYSTEM CONFIGURATION}

A typical smart farming system is composed of sensor node, control node, management decision supporting system, and integrated control system that is operated as a stand-alone system at a site as shown in Figure 1. This model focuses on the automation of farming process but requires non-unneglectable human intervention. Cloud service technologies can provide the next level of service from this model. Then, it requires equipment management system, data management system, model management system as well as Farm monitoring service and control service. The management service should be accessed in one-to-many (1: n) forms of virtualization between various devices and platforms. Furthermore, it should support information access with external public data and third-party models and application services. Unfortunately, this level of service is not provided in the real world but many related researches can be found and summarized in [24-25].

While this farming model in agricultural locations give values in production-marketing-service chain, the proposed smart urban farming model has many different aspects. We may enumerate several distinguishable characteristics of urban farming from agricultural farming as following;

1) While agricultural farming space has typical form with respect to agricultural plastic house standard, urban farming has no standard in size, form, and area.

2) Agricultural farming has outdoor facility gardening with area in average more than $7000 \mathrm{~m} 2$ but urban gardening has various sizes in that the average area size can be less than $100 \mathrm{~m} 2$ if the farmer uses personal space and much less size 9about $1000 \mathrm{~m} 2$ ) even if the public idle land is provided.

3) While agricultural farming is a family business and the land is exclusively occupied by the family, urban farming typically shares the public land.

Thus, the smart farming model for urban farming should be much more simplified form and flexible in configuration. In urban farming, still there are several types of gardening structure such as indoor-garden, rooftop garden, and box-type garden. With respect to such environmental differences, the smart farming solution should care for different managing aspects in the amount of light, $\mathrm{CO} 2$, wind, temperature, humidity etc. Meaning that one common management solution may not exist. 
Therefore we propose a smart urban farming model configuration with flexible equipment connections and simplified structures. In 2016, Telecommunication Technology Association (TTA) set a standard information and communication technology (ICT) model as shown in Figure 2.

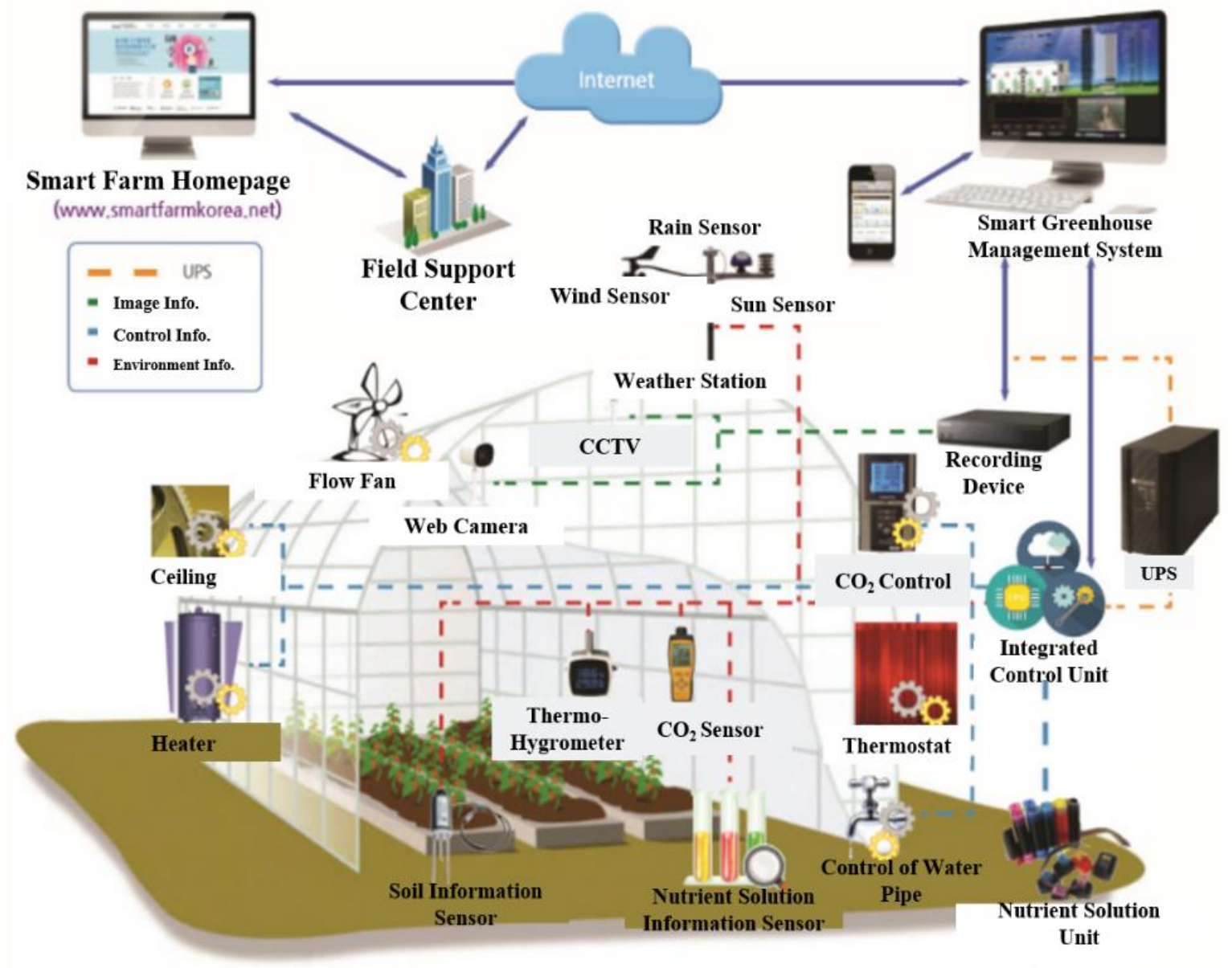

Figure 1. Typical existing smart farming model [23]

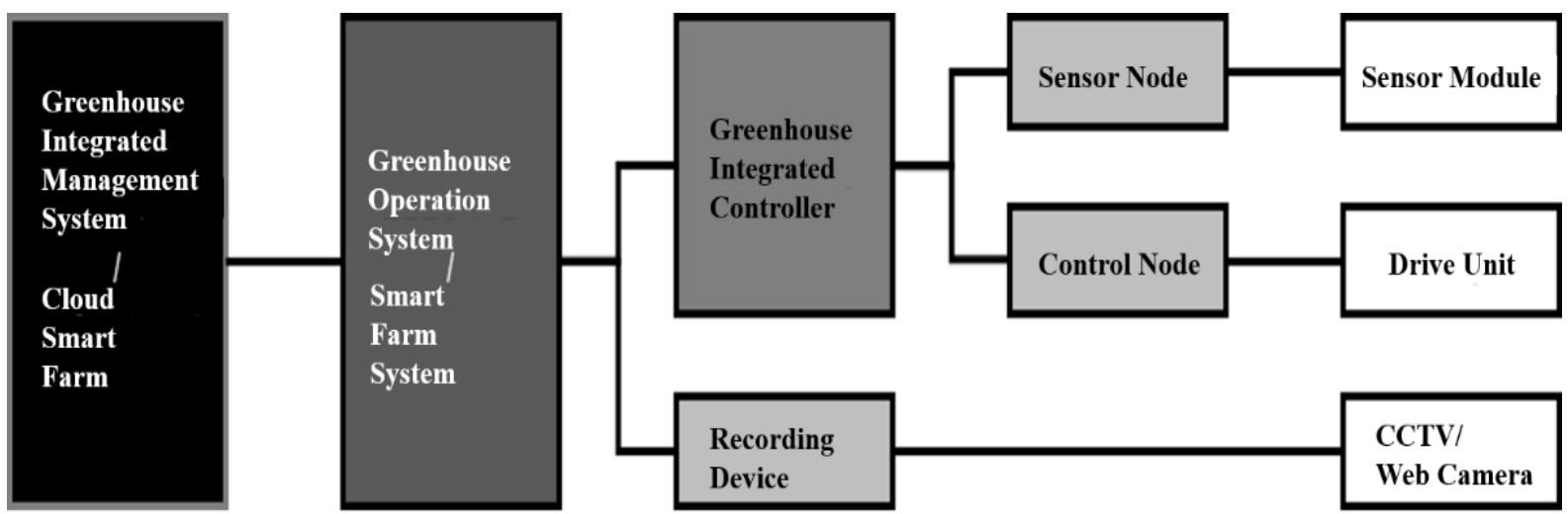

Figure 2. TTA standard for greenhouse ICT configuration 
With this standard, for ensuring the interoperability such as interface method and message exchange between each system component, the system should comply several standards such Greenhouse Control Data Standard (RUCFS-0009) and Smart Farm Greenhouse Control System Requirements Profile (TTAK.KO-06.0288).

However, the standard model shown in Figure 2 is based on the integrated control system architecture which has limited flexibility in component connections. In order to overcome that limitation, we adopt cloud service structure. Thus, we integrate greenhouse controller, sensor nodes, control nodes of the existing system into single 'smart farm integrated controller' under the standard specification (TTAK.KO06.0288) for interoperability) as shown in Figure 3.

Thus, the smart urban farming structure can be shown in Figure 4 in diagram as compared with the existing structure shown in Figure 2. The difference is the flexible connections of sensor components with adoption of the cloud system as explained in [23].

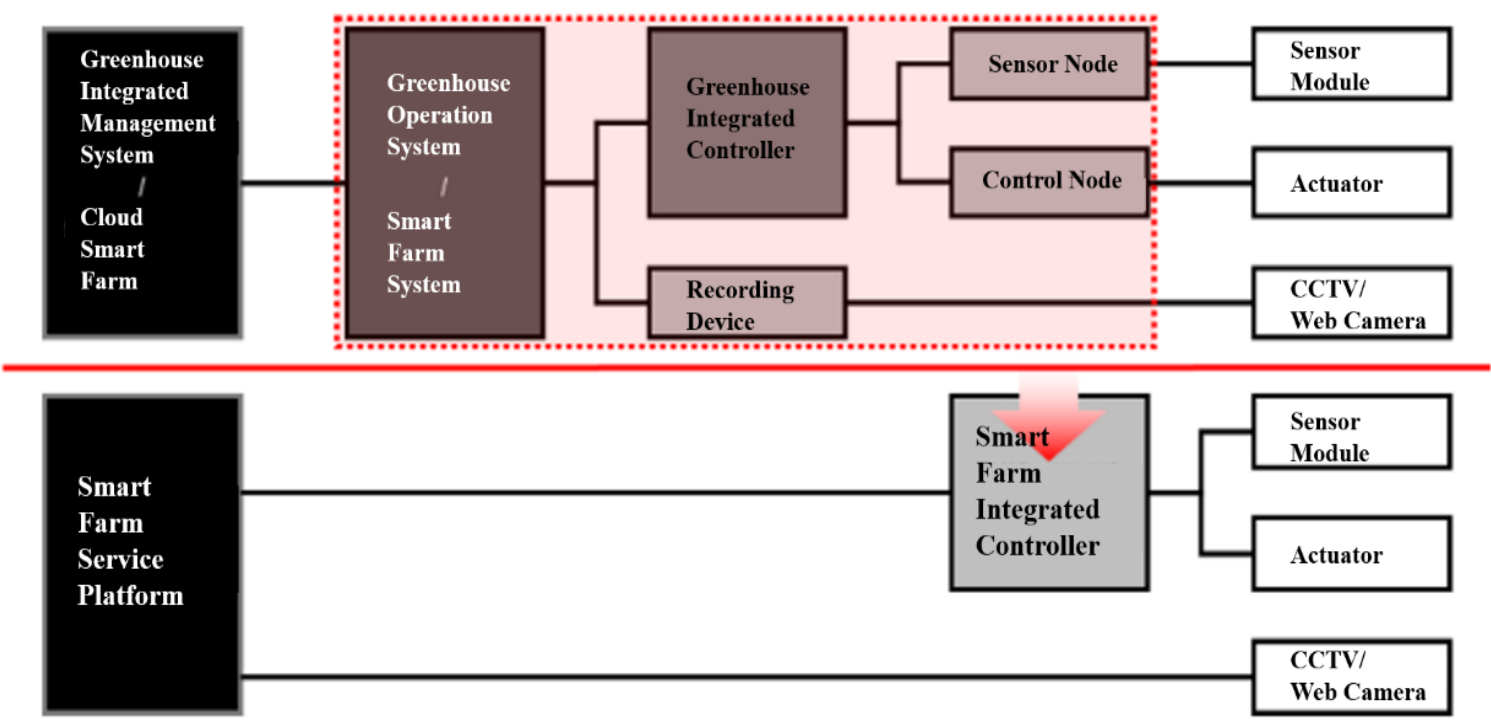

Figure 3. Proposed greenhouse configuration diagram

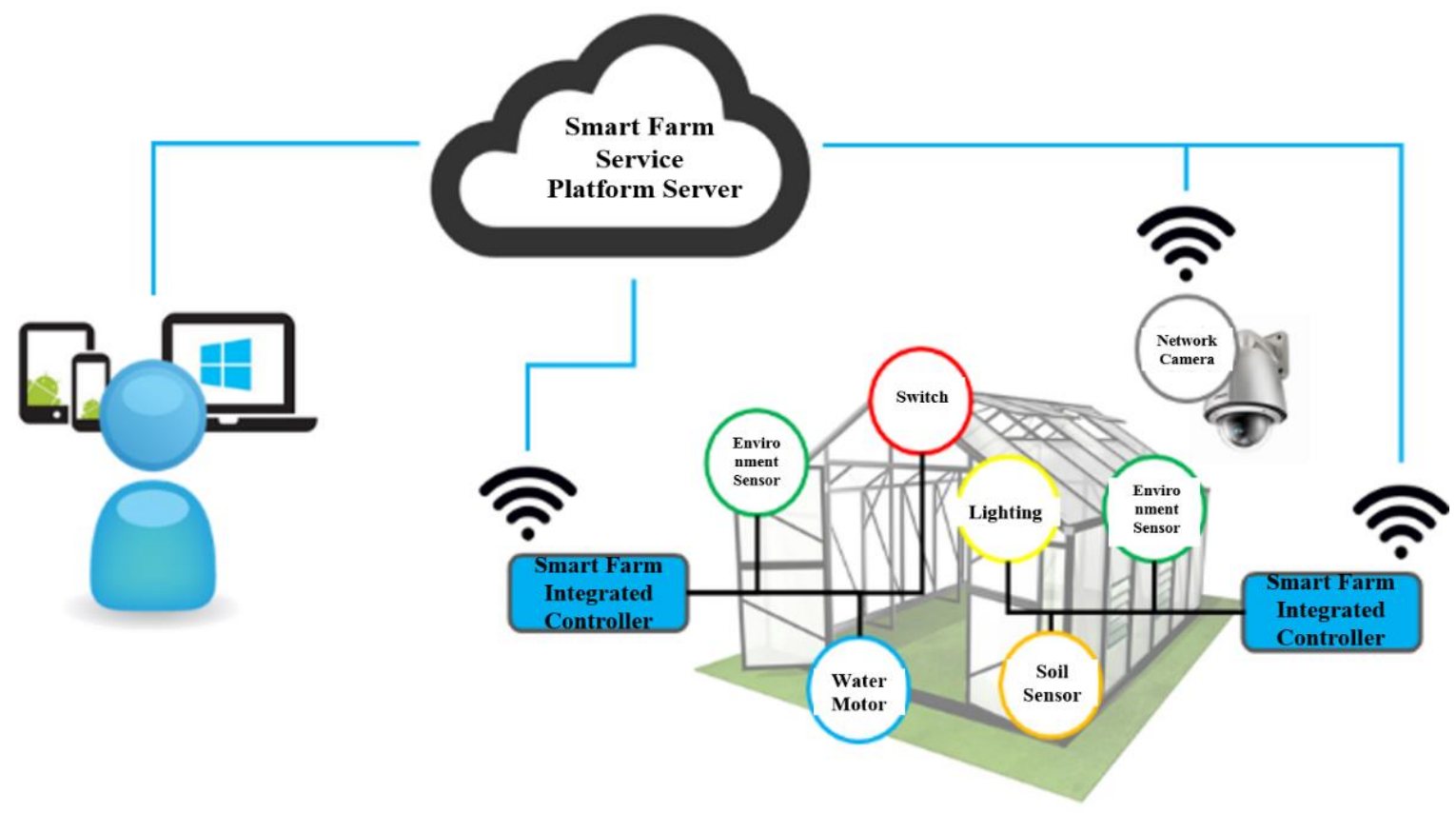

Figure 4. Proposed urban farming structure 
Thus, greenhouse management system communicates with the smart urban farming service platform under TTAK.KO-06.0288 part 4 and also communicate with integrated sensor nodes controller under TTAK.KO-06.0288 part 3. Smart urban farming service platform has the MQTT broker for integrated sensor nodes controller as shown in Figure 5.

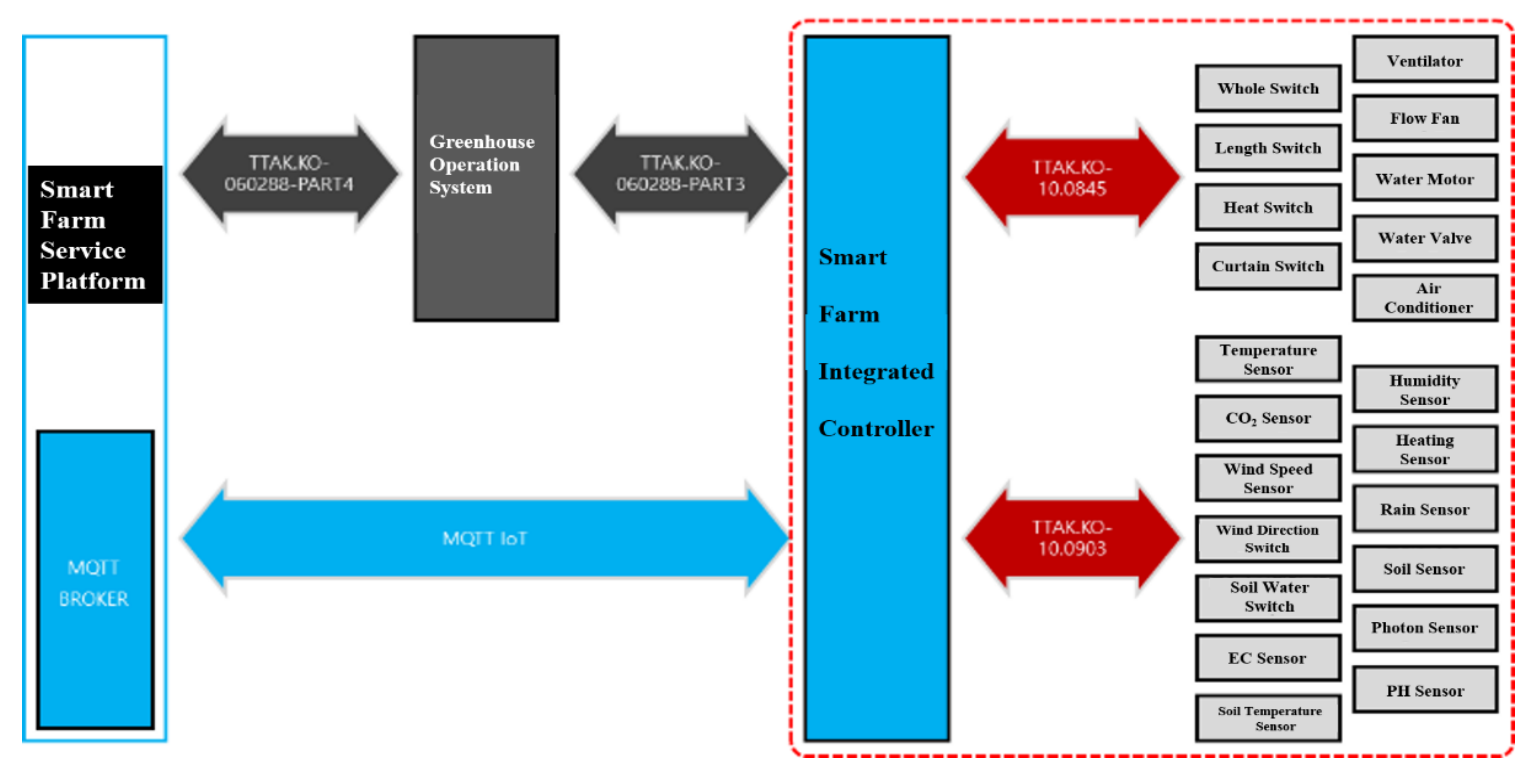

Figure 5. Connections in the proposed architecture

\section{COMPONENTS AND SERVICES}

The smart urban farming integrated controller (SUFIC) proposed in this paper supports the interlocking of all actuator equipment and sensor devices based on the TTA smart greenhouse standard. The hardware is designed to satisfy the standard specifications of smart greenhouse under driver interface driver interface (TTAK.KO-10.0845) and sensor interface (TTAK.KO-10.0903). Main controller constitutes STM32-32 bit microcontroller MCU module and provides $72 \mathrm{MHz}$ performance for fast interrupt response for real-time sensing and control. The hardware configuration of SUFIC can be summarized as shown in Figure 6.

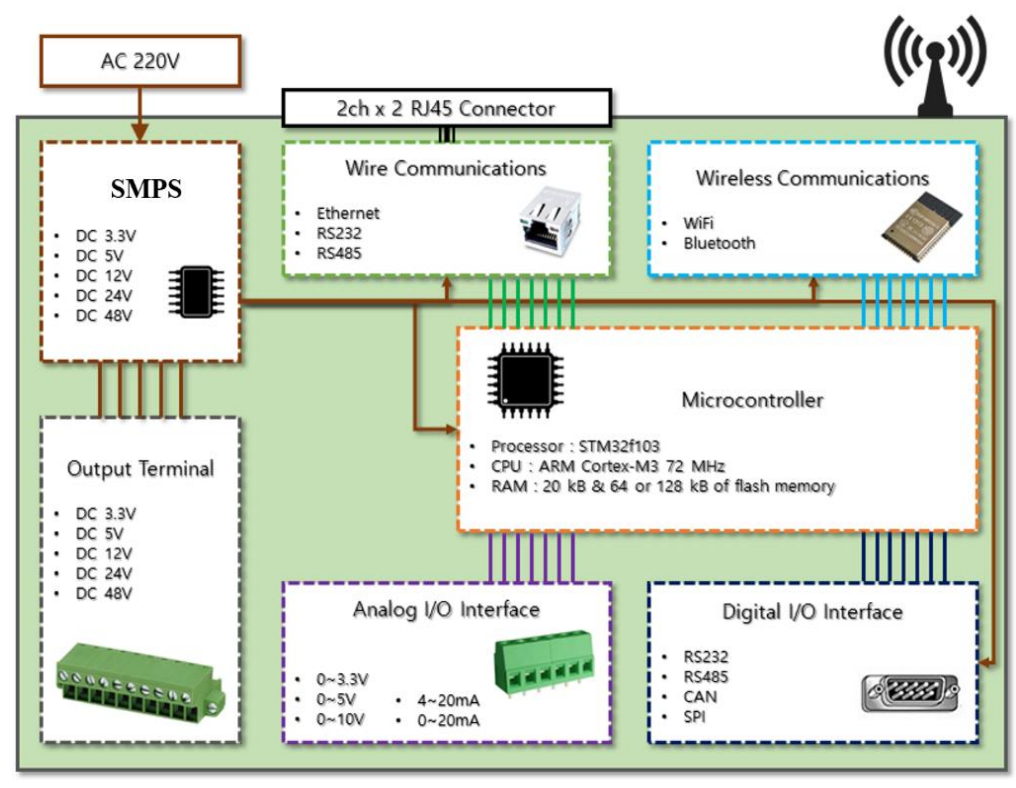

Figure 6. Hardware configuration of SUFIC

Indonesian J Elec Eng \& Comp Sci, Vol. 20, No. 1, October 2020 : 320 - 328 
Table 1. SUFIC message structure

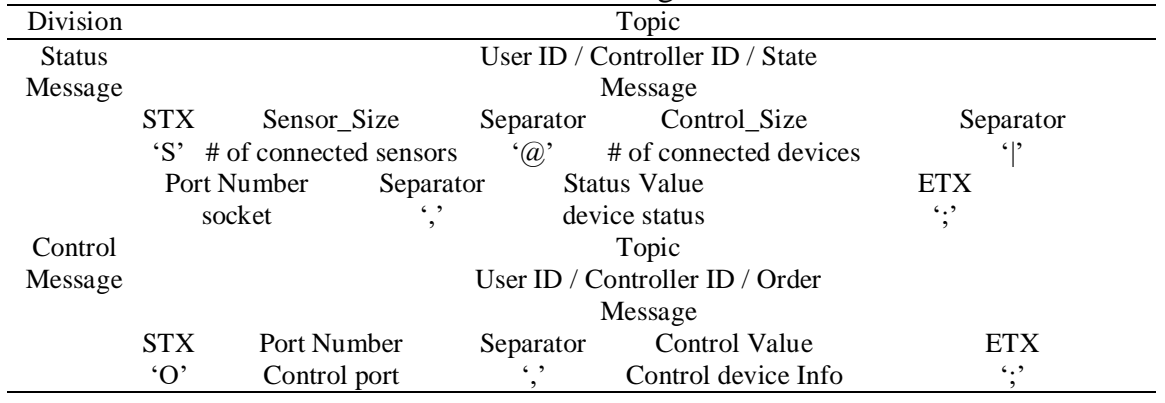

The standard MQTT message protocol of IoT is provided between SUFIC and service platform. We use user and controller information as a topic for MQTT communication to manage status / control information for each integrated controller device. It also classifies topic according to the function to distinguish status message and control message and defines protocol according to message standard between Smart Greenhouse Integrated Controller and Greenhouse Operation System. Table 1 shows the message structure of SUFIC. For monitoring and controlling devices, the proposed system uses MQTT protocol and HTTP API to enable the App services as shown in Figure 7.

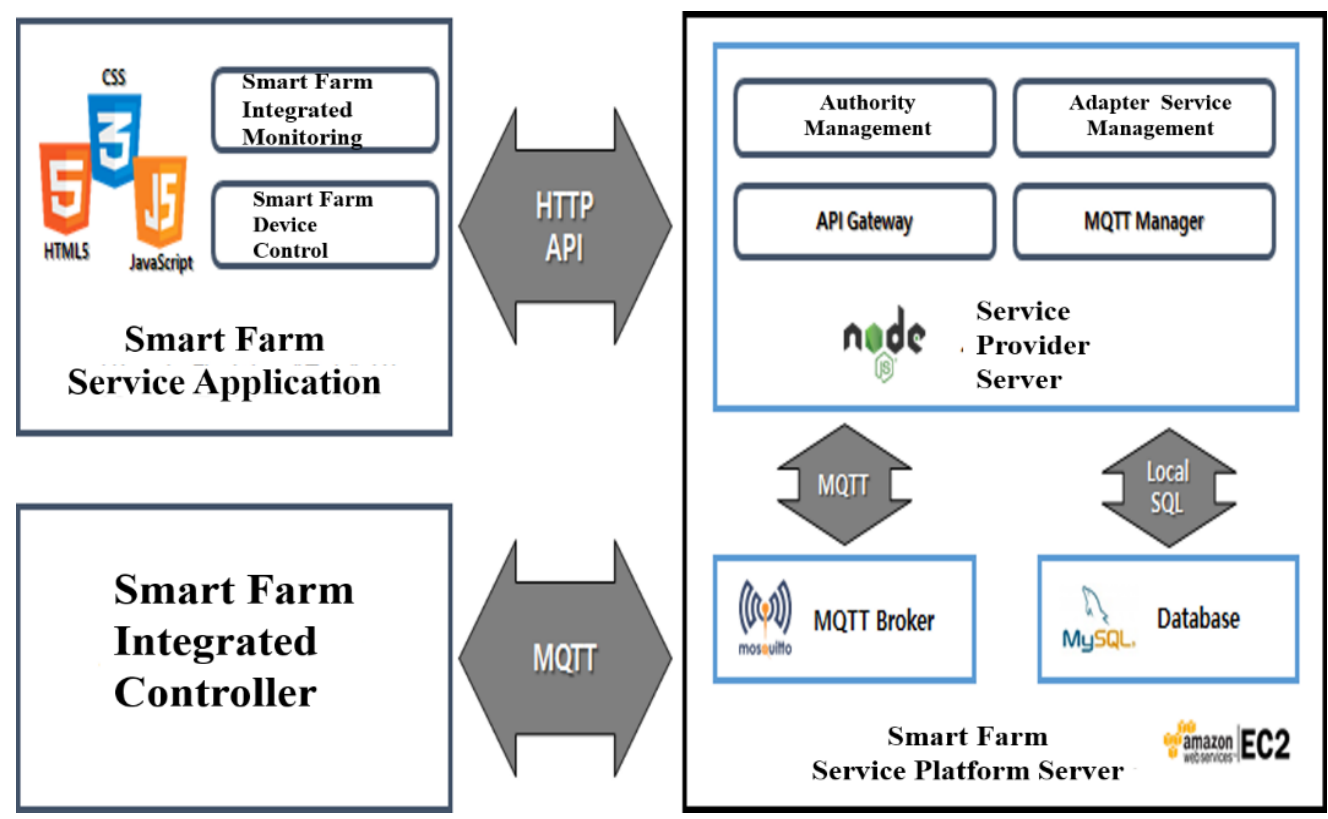

Figure 7. Modules of Service platform

In urban smart farm platform server, MQTT Broker for message exchange based on MQTT protocol and database for user and service information management are built in platform server based on web server environment. The service provision server connects to the MQTT Broker to receive sensor and device information published from the integrated controller, and provides the specified service according to the API request of the application. When a control request of a specific controller is received from the integrated management system, control data for the ID of the controller is transmitted to the corresponding controller through the MQTT Broker. In this way, unique ID is used for each integrated controller as a topic, allowing flexible monitoring and control services for each controller according to the configuration of the topic. Such platform server operations are summarized in Figure 8.

Service application to provide smart farming service is provided as web application using HTML / CSS / JavaScript and supports service in various client environments such as PC and mobile. Also, the system automatically builds components and interfaces for controller data received by the service platform (supports the ability to add / change components and interfaces according to user settings). Figure 9 and Figure 10 demonstrates an example of such additional services. 


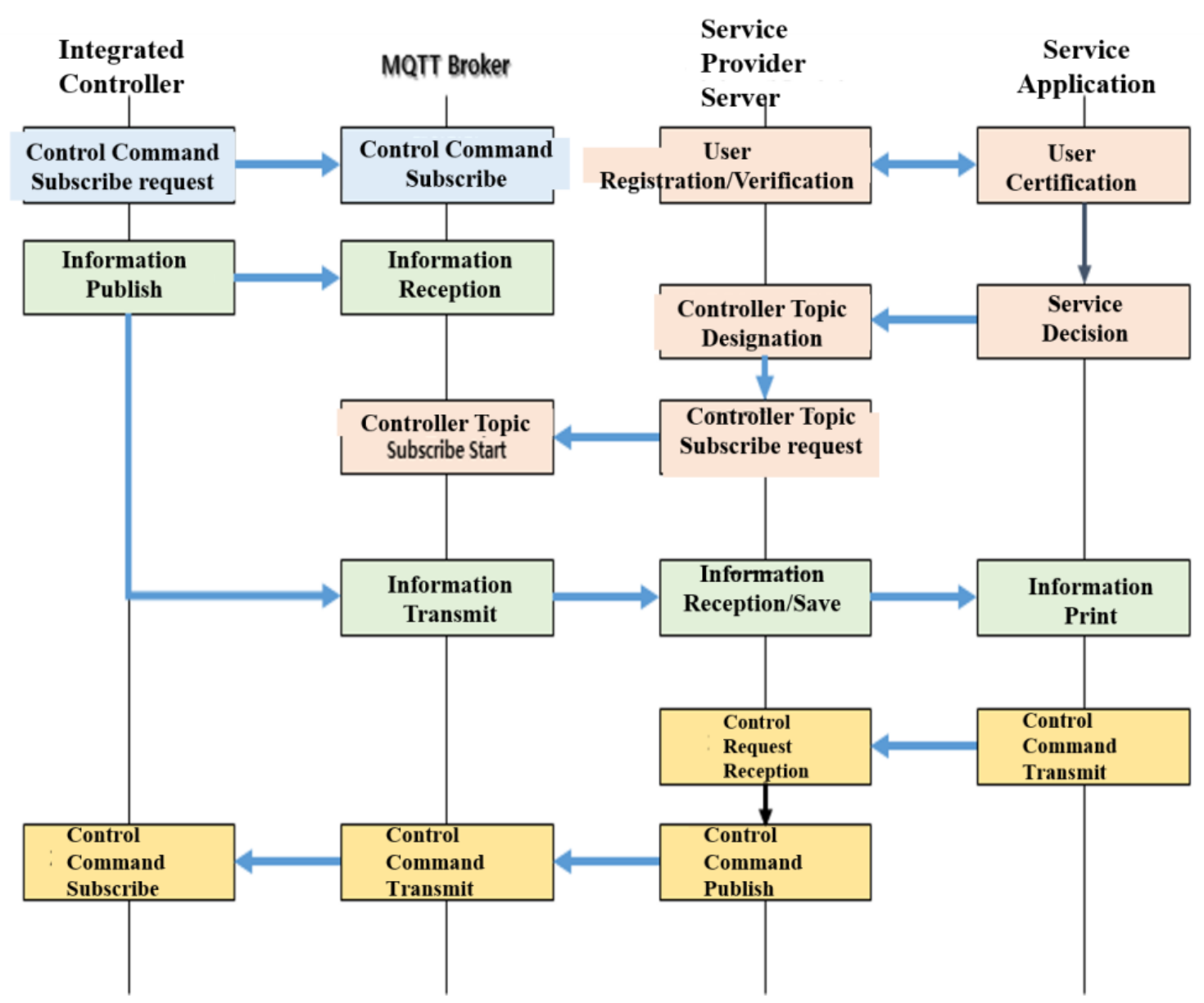

Figure 8. Platform server processing

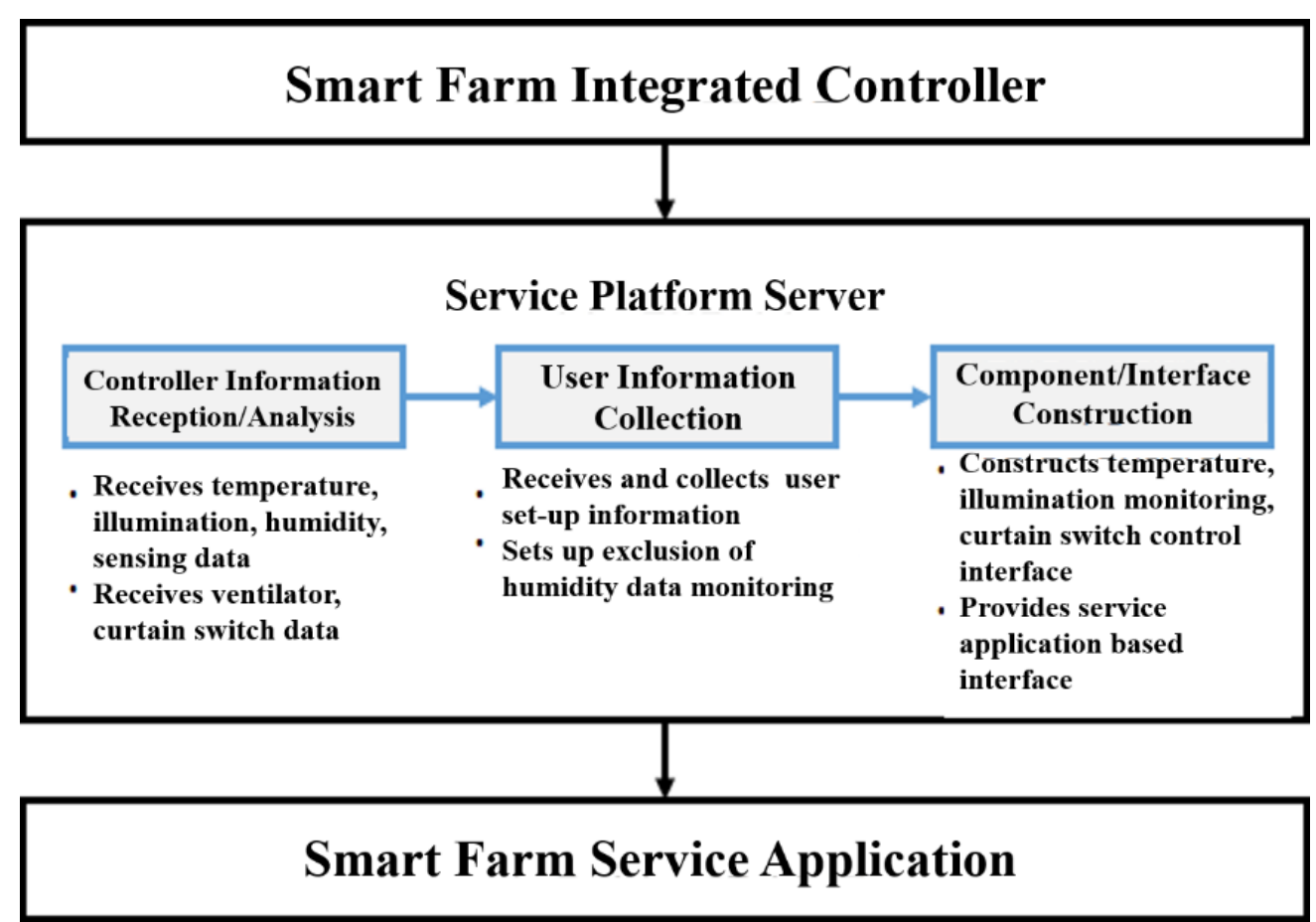

Figure 9. Example of service application supports 


\begin{tabular}{|c|c|c|c|c|}
\hline Master Controls & & Channels & & \\
\hline Pi Control & On & channel 1 & & \\
\hline Outputs & On & Control Value & 32 & \\
\hline Inputs Read & On & Setpoint Value & 40 & \\
\hline$\oplus$ More & & Action (\%) & 100 & \\
\hline$\theta$ Edit & & 4 Edit & & \\
\hline $\mathrm{Pi}$ Control & On & $\oplus$ Ouick Plot & & (109) \\
\hline Outputs & On & (4) channel 2 & & \\
\hline Inouts Read & n & (4) channel 3 & & \\
\hline & & (4) channel 4 & & \\
\hline Pi Control Freq & 1 & Channel Settings & & 0 \\
\hline Input Poll Freq & 1 & & & \\
\hline System Status & () & & & \\
\hline
\end{tabular}

Figure 10. Example of widget creation for automatic application service

\section{CONCLUSION}

Smart farming system using IoT supports becomes more popular worldwide. Especially, recent progress in this field owes to the cloud technology and big data application $[11,23]$. However, in Korea, there is another need for technology based agriculture system. Smart urban farming is different from production/marketing oriented smart agricultural farming in that it uses small shared area in cropping and the gardening condition is also different from usual outdoor farming in many aspects. Thus, we need to develop a new model of smart farming in urban environment such as rooftop and indoor gardening.

In this paper, we propose a cloud system based smart urban farming model that simplifies standard ICT greenhouse model established in 2016 by TTA. The hardware design of smart urban farming integrated controller is specified and subsequent service applications and message handling is explained. With this easyto-access but flexible-in-connecting sensors and equipment model, smart urban farming is expected to be more popular and thus to contribute to the national policy to enhance the technology in next five years.

\section{ACKNOWLEDGEMENTS}

This work was supported by the BB21+ Project in 2019.

\section{REFERENCES}

[1] A. McBratney, et al., "Future Directions of Precision Agriculture," Journal of Precision Agriculture, vol. 6, pp. 7-23, 2011.

[2] N. Gershenfeld, et al., "The Internet of Things," Scientific American, vol. 291, pp. 76-81, 2011.

[3] A. Kamilaris, et al., "Agri-IoT: A semantic framework for Internet of Things-enabled smart farming applications," 2016 IEEE 3rd World Forum on Internet of Things, pp. 442-447, 2016.

[4] F. Balducci, et al., "Machine learning applications on agricultural datasets for smart farm enhancement," Machines, vol. 6, pp. 1-22, 2018.

[5] A. Madushanki, et al., "Adoption of the Internet of Things (IoT) in Agriculture and Smart Farming towards Urban Greening: A Review," International Journal of Advanced Computer Science and Applications, vol. 10, pp. 11-28, 2019.

[6] R. Venkatesan and A. Tamilvanan, "A sustainable agricultural system using IoT," in International Conference on Communication and Signal Processing, pp. 763-767, 2017. 
[7] K. Taylor, et al., "Farming the web of things," IEEE Intelligent Systems, vol. 28, pp. 12-19, 2013.

[8] S. Vaishali, et al., "Mobile integrated smart irrigation management and monitoring system using IOT," in International Conference on Communication and Signal Processing, pp. 2164-2167, 2017.

[9] M. Rajkumar, et al., "Intelligent irrigation system - An IOT based approach," in International Conference on Innovations in Green Energy and Healthcare Technologies, pp. 1-5, 2017.

[10] A. Rau, et al., "IoT based smart irrigation system and nutrient detection with disease analysis," in IEEE Region 10 Symposium, pp. 1-4, 2017.

[11] S. Salvi, et al., "Cloud based data analysis and monitoring of smart multi-level irrigation system using IoT," in International Conference on IoT in Social, Mobile, Analytics and Cloud, pp. 752-757, 2017.

[12] Karl Aberer, Manfred Hauswirth, and Ali Salehi. "A middleware for fast and flexible sensor network deployment," in Proceedings of the 32nd international conference on Very large data bases, pp. 1199-1202, 2006.

[13] Michael Compton, et al., "The SSN ontology of the W3C semantic sensor network incubator group," Science, Services and Agents on the World Wide Web, vol. 17, pp. 25-32, 2012.

[14] C. Nwabueze, et al., "Enhancing Agricultural Production Using Wireless Sensor Network," Iconic Research And Engineering Journal, vol. 2, pp. 274-284, 2019.

[15] C. Yoon, et al., "Implement smart farm with IoT technology," in 2018 20th International Conference on Advanced Communication Technology, pp. 749-752, 2018.

[16] Y. Waché, et al., "Prospects for food fermentation in South-East Asia, topics from the tropical fermentation and biotechnology network at the end of the AsiFood Erasmus+ Project," Frontiers in microbiology, vol. 9, 2018.

[17] C. Dupont, et al., "An open IoT platform to promote eco-sustainable innovation in Western Africa: real urban and rural testbeds," Wireless Communications and Mobile Computing, vol. 2018, pp. 1-17, 2018.

[18] B. Y. Kim, et al., "Top 10 Issues of Agricultural Policies, Focus in Agricultural Policy," published by Korea Rural Economic Institute, vol. 142, pp 1-27, 2017.

[19] M. Bailkey and J. Nasr, "From Brownfields to Greenfields: Producing Food in North American Cities," Community Food Security News, 2000.

[20] T. G. Kim, et al., "Community Business Development Plan”, Korea Rural Economic Institute, pp. 1-189, 2012.

[21] I. K. Oh, et al., "Perspectives on the Potential of Job Creation Based on Participation Motivation, Attitudes, and Barriers to Urban Farming: Focused on Young Farmers," Journal of The Korean Regional Development Association, vol. 31, pp. 55-70, 2019.

[22] I. B. Lee, et al., "Analysis of Research Trend and Core TechnologiesBased on ICT to Materialize Smart-farm," Protected Horticulture and Plant Factory, vol. 25, No. 1, pp. 30-41, 2016.

[23] S. Y. Lee, "Smart Farm Technology based on Cloud," Journal of The Korean Institute of Communication Sciences, vol. 34, no. 1, pp. 51-57, 2016.

[24] S. Wolfert, et al., "Big data in smart farming-a review," Agricultural Systems, vol. 153, pp. 69-80, 2017.

[25] J. Ji, et al., "Collaborative Filtering Algorithm Based on User-Item Attribute Preference," Journal of information and communication convergence engineering, vol. 17, no. 2, pp. 135-141, 2019.

\section{BIOGRAPHIES OF AUTHOR}

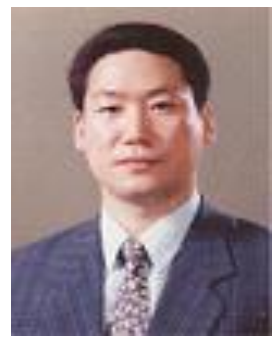

Am suk Oh received the B.S. and M.S. degrees in computer science from Busan National University and Chung-ang University, respectively. He received Ph.D degree at the computer engineering of Busan National University. He is currently with the Department of Digital Media Engineering, Tongmyong University as Professor. His research interests are Database, Healthcare System, Big Data and IoT. 